\title{
Comparison of Short and Mid-Term Mortality and Morbidity in Patients With Severe Aortic Stenosis (Intermediate/High Risk) Who Underwent Transcatheter Aortic Valve Replacement and Surgical Aortic Valve Replacement
}

\author{
Mehmet Ali Yesiltas, MD, ${ }^{1}$ İsmail Haberal, $\mathrm{MD},{ }^{2}$ Yusuf Kuserli, MD,${ }^{1}$ Ahmet Yildiz, MD, ${ }^{3}$ \\ Ahmet Ozan Koyuncu, MD, ${ }^{2}$ Sadiye Deniz Özsoy, MD $^{2}$ \\ ${ }^{1}$ Department of Cardiovascular Surgery, Health Ministry Bakırköy Dr. Sadi Konuk Training and Research Hospital, İstanbul, Turkey; \\ ${ }^{2}$ Department of Cardiovascular Surgery, İstanbul University Cerrahpasa Institute of Cardiology, İstanbul, Turkey; ${ }^{3}$ Department of \\ Cardiology, İstanbul University Cerrahpasa Institute of Cardiology, İstanbul, Turkey
}

\section{ABSTRACT}

Treatment protocols for severe aortic valve stenosis include surgical aortic valve replacement (SAVR), balloon valvuloplasty, transcatheter aortic valve replacement (TAVR), and medical treatment. Because the success rates are getting higher with both SAVR and TAVR, making the right treatment decision is important.

This study retrospectively shows the short- (1 month) and mid-term (6 months) mortality and morbidity rate differences between 2 groups of patients, who arrived to our hospital from January 2014 through October 2018. The first group consists of 54 patients who underwent mid-high risk SAVR operations at Istanbul University-Cerrahpasa, Institute of Cardiology, Department of Cardiovascular Surgery. The second group consists of 57 patients who underwent TAVR at the Cardiology Department.

Preoperative evaluation showed that the mean age of the SAVR group (71.5 years) was higher than the TAVR group (80 years). Also, the history of previous cardiac valve replacement surgery significantly was higher in the SAVR group than the TAVR group $(P=.028)$. There were no significant differences between the remaining preoperative tests and diagnostic procedures.

Of the patients who underwent SAVR, 3.7\% experienced postoperative cardiac arrhythmias, while the $17.5 \%$ of patients from the TAVR group experienced cardiac arrhythmias after the procedure. This difference between the groups were statistically significant. Mortality rate was $9.3 \%$ in the SAVR group and $5.3 \%$ in the TAVR group. The mortality rate was not statistically different between the groups. There was no significant difference between the groups in the means of neurological incidents. The TAVR group had more vascular complications $(17.9 \%$ to none) and pacemaker implantations $(21.4 \%$ to $1.9 \%)$.

Received February 6, 2020; accepted March 20, 2020.

Correspondence: Mehmet Ali YEŞiLTAŞ, Consultant, Department of Cardiovascular Surgery, S.B.Ü. Bakırköy Dr. Sadi Konuk E.A.H., Zuhuratbaba Mah. No:11; +90-212- 4147171; fax: +90-212-414-64-94 (e-mail:dr:maliyes@gmail.com).
Minor or major bleeding was the most common reason for admission to the hospital after SAVR. Seven out of 10 patients experienced bleeding. Aortic regurgitation was more common in the TAVR group at the first and sixth month following the procedure. Ratios between the gradient values were higher in the SAVR group $(P<.001)$. Peak gradient values at the sixth month following the procedure were lower than the values of the first month $(P<.040)$.

Aortic regurgitation symptoms increased with patients at the mid-term follow-up appointment. To prevent the vascular complications in the TAVR group, preoperative peripheral vascular examination thoroughly should be performed. Considering that bleeding disorders are the main reason the SAVR group arrived to the hospital, INR values should closely be monitored. There seems to be no mortality difference between the groups at the six-month follow up, but studies should continue with more patients and long-term results.

\section{INTRODUCTION}

Aortic valve stenosis is a common cardiac problem. Treatment protocols for severe aortic valve stenosis include surgical aortic valve replacement (SAVR), balloon valvuloplasty, transcatheter aortic valve replacement (TAVR), and medical treatment. Since the success rates are getting higher among the well adapted SAVR and the lately popular TAVR, making the right decision between these treatment options is important [Nishimura 2002]. Surgical aortic valve surgery improves survival and symptoms, but increases the risk of operation complications and death in some patient groups (low left ventricular function, concomitant diseases) [Smith 2011].

\section{METHODS}

Patients: Between January 2014 through October 2018, we enrolled 111 patients with intermediate/severe aortic stenosis and cardiac symptoms at Istanbul UniversityCerrahpasa Institute of Cardiology. According to echocardiography results, severe aortic stenosis was found with an 
Table 1. Characteristics of the Patients at Baseline

\begin{tabular}{|c|c|c|c|c|c|}
\hline Male & 26 & 48.1 & 24 & 42.1 & .522 \\
\hline Female & 28 & 51.9 & 33 & 57.9 & .522 \\
\hline \multicolumn{6}{|l|}{ Diabetes Mellitus } \\
\hline No & 30 & 55.6 & 28 & 49.1 & .498 \\
\hline Yes & 24 & 44.4 & 29 & 50.9 & .498 \\
\hline \multicolumn{6}{|c|}{ Chronic Obst. Lung Disease } \\
\hline No & 45 & 83.3 & 46 & 80.7 & .718 \\
\hline Yes & 4 & 7.4 & 3 & 5.3 & .712 \\
\hline \multicolumn{6}{|c|}{ Cerebrovascular Disease } \\
\hline No & 51 & 94.4 & 53 & 93.0 & 1 \\
\hline Yes & 3 & 5.6 & 4 & 7.0 & 1 \\
\hline \multicolumn{6}{|c|}{ Previous Cardiac Operation } \\
\hline No & 41 & 75.9 & 45 & 78.9 & \\
\hline CABG & 4 & 7.4 & 10 & 17.5 & .028 \\
\hline Valve operation & 9 & 16.7 & 2 & 3.5 & \\
\hline \multicolumn{6}{|l|}{ Previous $\mathrm{PCl}$} \\
\hline \multicolumn{6}{|l|}{ Heart Failure } \\
\hline No & 45 & 83.3 & 50 & 87.7 & \\
\hline Yes & 9 & 16.7 & 7 & 12.3 & .511 \\
\hline \multicolumn{6}{|l|}{ Hypertension } \\
\hline No & 8 & 14.8 & 4 & 7 & \\
\hline Yes & 45 & 85.2 & 53 & 93 & .186 \\
\hline \multicolumn{6}{|l|}{ Atrial Fibrillation } \\
\hline No & 45 & 85.2 & 46 & 80.7 & \\
\hline Yes & 8 & 14.8 & 11 & 19.3 & .531 \\
\hline \multicolumn{6}{|c|}{ New York Heart Association (NYHA) Classification } \\
\hline Class 3 & 19 & 35.2 & 17 & 29.8 & \\
\hline Class 4 & 35 & 64.8 & 40 & 70.2 & .546 \\
\hline \multicolumn{6}{|l|}{ Dyspnea } \\
\hline No & 5 & 9.3 & 4 & 7 & .738 \\
\hline Yes & 49 & 90.7 & 53 & 93 & \\
\hline
\end{tabular}


Table 1. Characteristics of the Patients at Baseline [Cont.]

\begin{tabular}{|c|c|c|c|c|c|}
\hline No & 50 & 92.6 & 53 & 93 & 1 \\
\hline Yes & 4 & 7.4 & 4 & 7 & \\
\hline Mean Gradient & $47.74 \pm 13.5$ & $49.11 \pm 14.74$ & 0.613 & & \\
\hline \multicolumn{6}{|l|}{ STS } \\
\hline$<8$ & 21 & 38.9 & 10 & 17.5 & .012 \\
\hline$>8$ & 33 & 61.1 & 47 & 82.5 & .012 \\
\hline
\end{tabular}

Chi-square was applied.

aortic valve area $<1 \mathrm{~cm}^{2}$, mean gradient $>40 \mathrm{mmHg}$, and/ or peak flow velocity $>4 \mathrm{~m} / \mathrm{s}$. Patients were considered to be at intermediate and high risk on the basis of clinical assessments by a multidisciplinary heart team, which used a guideline based on a risk model developed by the Society of Thoracic Surgeons (STS) to estimate the risk of death at 30 days after surgery [O'Brien 2009]. Patients excluded from the study were pediatric patients ( $<18$ years), those with coronary artery disease, patients with severe aortic insufficiency, those with moderate-to-severe mitral insufficiency, and dialysisdependent patients.

Device and procedure: For TAVR, the balloon expandable Corevalve aortic heart valve system $(26 \mathrm{~mm}, 29 \mathrm{~mm}$, or $31 \mathrm{~mm})$ (Medtronic ${ }^{\circledR}$, USA) was applied to patients with a transfemoral approach. All patients underwent balloon dilatation before and after the procedure. Patients received aspirin (100mg) and clopidogrel $(300 \mathrm{mg})$ before the procedure and heparin during the procedure. During the procedure, 5000 IU heparin is applied first, and ACT $>250$ is expected. If ACT $>250$ is not available, heparin is administered as an additional dose. Patients continued to take aspirin $(100 \mathrm{mg})$ and clopidogrel $(75 \mathrm{mg})$ postoperatively for the first 6 months.

For SAVR, median sternotomy was performed to start the procedure to implant the mechanical aortic valve (Medtronic ${ }, \mathrm{USA}$ ). In the SAVR procedure, 350 units $/ \mathrm{kg}$ heparin is made before cardiopulmonary bypass and ACT $>450$ is expected. Cardiopulmonary bypass was performed after aortic-atrial cannulation. Cardiopulmonary bypass was achieved using a membrane oxygenator and roller pump, with an average flow of $2 \mathrm{LT} / \mathrm{min} / \mathrm{m} 2$ and an average perfusion pressure of $70 \mathrm{mmHg}$. Patients were cooled to $28^{\circ} \mathrm{C}$. After cross clamping, isothermal potassium rich blood cardioplegia was given antegrad (2/3 of them directly in coronary ostiums) and retrograd(1/3 of them in coronary sinus). Following transverse aortotomy, the aortic valve was reached; the aortic valve with stenosis was excised and calcifications thoroughly were cleaned. While $19 \mathrm{~mm}$ of mechanical valves cause serious residual gradient, $21 \mathrm{~mm}$ covers provide adequate hemodynamic improvement in those with a body surface area of
1.5-1.7 $\mathrm{m}^{2}$. For this reason, the largest cover selection was made in accordance with the body surface area whenever possible. In addition, aortic annulus measurement was performed with a valve, and the valve was implanted during the surgery. The prosthetic valve was sutured with $2 / 0$ polyester pleated sutures. Cardiopulmonary bypass was terminated, and the procedure was done. Although it is thought to be more appropriate to place the biological valve in some patients, mechanical valve was applied as required by our hospital, during the study.

During the trial, transthoracic echocardiography (TTE) was performed with GE Healthcare Vivid ${ }^{\mathrm{TM}}$ T8 at 1 and 6 months preoperatively and postoperatively in all patients undergoing TAVR and SAVR. Ejection fraction, aortic valve diameter, mean and peak gradient in the aorta, and paravalvular leak were evaluated with TTE from baseline to 6 months.

Trial design: The aim of this study was to evaluate the mortality and morbidity rates of patients with moderate to high risk of severe aortic stenosis after 1 and 6 months, following transcatheter aortic valve replacement and surgical aortic valve replacement. The treatment methods were formed, according to the decision of the cardiac team. The heart team included the cardiovascular surgeon, interventional cardiologist, primary cardiologist, and anesthesiologist. There were no subgroups in patients who underwent TAVR or SAVR. All patients were classified, according to Society of Thoracic Surgeons scoring. Risk scores were calculated online using the official website (http://riskcalc.sts.org/stswebriskcalc/ calculate). Our patients were invited for control at the first and sixth months, following SAVR and TAVR for echocardiography, according to hospital protocol. In addition, ECO results at the first and sixth months also were recorded. Color Doppler evaluation has performed just below the valve for paravalvular jets, and at the coaptation point of the leaflets for central regurgitation. Whenever possible, the quantification of the prosthetic regurgitant volume, effective regurgitant orifice area, and regurgitant fraction were performed.

End points: Patients received follow up for 6 months. During this period, death of any cause was considered the first end point. Major or minor cerebrovascular events 
Table 2.

\begin{tabular}{|c|c|c|c|c|c|}
\hline No & 53 & 98.1 & 54 & 94.7 & $.619 *$ \\
\hline Yes & 1 & 1.9 & 3 & 5.3 & $.619 *$ \\
\hline No & 52 & 96.3 & 47 & 82.5 & .019 \\
\hline Yes & 2 & 3.7 & 10 & 17.5 & .019 \\
\hline \multicolumn{6}{|l|}{ Death } \\
\hline No & 49 & 90.7 & 54 & 94.7 & $.482 *$ \\
\hline Yes & 5 & 9.3 & 3 & 5.3 & \\
\hline \multicolumn{6}{|l|}{ Rehospitalization } \\
\hline No & 42 & 80.8 & 33 & 58.9 & \\
\hline First week & 4 & 7.7 & 4 & 7.1 & .052 \\
\hline First week-first month & 3 & 5.8 & 9 & 16.1 & \\
\hline First-sixth months & 3 & 5.8 & 10 & 17.9 & \\
\hline \multicolumn{6}{|l|}{ Cerebrovascular Event } \\
\hline No & 53 & 98.1 & 54 & 94.7 & $.619 *$ \\
\hline Yes & 1 & 1.9 & 3 & 5.3 & \\
\hline \multicolumn{6}{|c|}{ New Permanent Pacemakers } \\
\hline No & 53 & 98.1 & 44 & 78.6 & $<.001$ \\
\hline Yes & 1 & 1.9 & 12 & 21.4 & $<.001$ \\
\hline
\end{tabular}

Chi-Square test was applied.*Fisher's exact test

(major cerebrovascular accident is bed-dependent or death; a minor cerebrovascular event is a temporary ischemic attack or motor motion defect, which is a return), rehospitalization (condition requiring rehospitalization for any reason), acute renal failure, vascular complication, major or minor hemorrhage (major bleeding was defined as whether there was a need for reoperation), and newly developing atrial fibrillation were evaluated as secondary outcomes. Clinical symptoms of each patient at the first and sixth months of follow up followed NYHA classification. NYHA was used to standardize patient cardiac symptoms. According to NYHA Classification - The Stages of Heart
Failure, Class I involves no symptoms and no limitation in ordinary physical activity, e.g. shortness of breath when walking, climbing stairs etc.; Class II involves mild symptoms (mild shortness of breath and/or angina) and slight limitation during ordinary activity; Class III features marked limitation in activity due to symptoms, even during less-than-ordinary activity, e.g. walking short distances (50-100 m). Patients are comfortable only at rest; Class IV involves severe limitations. Patients experience symptoms even while at rest, and patients mostly are bedbound; and Class V includes patients whose NYHA class is not listed or unable to determine. 
Table 3. Comparison of clinical features of AVR and TAVI patients with postop 1st-6th month NYHA symptoms

\begin{tabular}{|c|c|c|c|c|c|}
\hline Özellik & $\mathrm{N}$ & $\%$ & $N$ & $\%$ & $P$ \\
\hline 1 & 30 & 62.5 & 13 & 24.1 & \\
\hline 2 & 18 & 37.5 & 24 & 44.4 & $<.001$ \\
\hline 4 & 0 & 0 & 1 & 1.9 & \\
\hline \multicolumn{6}{|c|}{ NYHA (Sixth month) } \\
\hline 1 & 44 & 91.7 & 18 & 34.0 & $<.001$ \\
\hline 2 & 4 & 8.3 & 22 & 41.5 & \\
\hline 3 & 0 & 0 & 13 & 24.5 & \\
\hline
\end{tabular}

Chi-Square test was applied. *Fisher's exact test

Paravalvular leakage and mean gradient were evaluated at 1 month and 6 months with transthoracic echocardiography.

Statistical analysis: Data were analyzed using SPSS 21.0 software package. Numerical data were expressed as mean \pm standard deviation and median (minimum-maximum). Categorical data were expressed in frequency $(\mathrm{N})$ and percentage (\%). Chi-square test between categorical variables was used to evaluate the difference between the groups. Fisher's exact test was used in cases where Chi-square test assumptions were not used. Kolmogorov Smirnoff test was used to evaluate the normal distribution for numerical data. Comparison of continuous variables between 2 independent groups, if normal distribution conditions are provided; $\mathrm{t}$ test was used. Mann Whitney $U$ test was used in cases, where normal distribution conditions could not be achieved. The Wilcoxon test was used to compare the non-normally distributed continuous variables of 2 dependent groups. A $P$ value of $<.05$ was accepted for statistical significance (Table 1).

\section{RESULTS}

Patients: Among the 111 patients who underwent randomization, 57 were assigned to TAVR and 54 to SAVR. Of the SAVR group population, $48.1 \%$ was male; with the TAVR group, $42.1 \%$ was male. There was no statistically significant difference between the 2 groups, in terms of gender. The median age of patients in the SAVR group was 71.5 years (18-85), while the median age of the patients in the TAVR group was 80 years (49-94). The median age of the TAVR group was statistically significantly higher than that of the SAVR group. The TAVR group included more clinically fragile patients. Patients were followed up on for 6 months in both groups. The characteristics of the patients at baseline were well balanced into the 2 trial groups. Although there was an equal distribution between STS $>8$ in both groups, and the $P$ value was significant between $\mathrm{STS}<8$. Of the SAVR group, $75.9 \%$ had no cardiac operations, 7.4\% had CABG, and $16.7 \%$ had a valve operation. In the TAVR group, $78.9 \%$ had no operation, $17.5 \%$ had a CABG operation, and $3.5 \%$ had a history of valve operation. The history of valve operation significantly was higher in the SAVR group compared with the TAVR group $(P=.028)$. The reason for this situation is that TAVR cannot be applied in the previous SAVR patient group. There is no significant difference in other preoperative comparisons. The preoperative basic clinical features for the treated patients are shown in Table 1 .

Procedure outcomes: death, stroke, and other clinical endpoints.

A total of 8 patients $(7.2 \%$; 5 patients in the SAVR group and 3 patients in TAVR group) died either during the procedure or within 5 days following surgery. Two of the patients suffered from cardiac arrhythmic death, following arrhythmia (ventricular fibrillation) during the procedure. The other patient had sudden cardiac death at home in the postoperative third month. After the procedure (2-4 days), the SAVR patients who died suffered multiorgan failure. There was no significant difference between the 2 groups, in terms of death. None of the patients developed valve embolization and thrombosis.

There was no significant difference in the primary end point of death from any cause or disabling stroke at 6 months between the TAVR group and the surgery group (in the TAVR group, $5.3 \%$; in the SAVR group, $1.9 \%)$. Patients in the SAVR group postoperatively were awakened hemiplegic and thought to be due to embolism in cardiopulmonary bypass. In the TAVR group, 1 in 3 patients immediately became hemiplegic following surgery. One of the other 2 patients had a temporary ischemic attack at the first week and the other at the sixth week. There was an ischemic cerebrovascular accident in the etiology of all patients.

The time-dependent effects of disabling stroke, lifethreatening bleeding, acute kidney injury, and major vascular complications all were significantly associated with 
Table 4. Comparison of preop, first month and sixth month ECO results in the SAVR and TAVR groups

\begin{tabular}{lccccccccccccc}
\hline & \multicolumn{3}{c}{ SAVR } & & & & \multicolumn{2}{c}{ TAVR } \\
& N & Mean \pm Std & Median & Min & Max & N & Mean \pm Std & Median & Min & Max & $P$ & \\
\hline Aortic Mean Gradient preoperative & 54 & $47.74 \pm 13.50$ & 46 & 16 & 83 & 57 & $49.11 \pm 14.74$ & 45 & 24 & 110 & $<.613$ \\
Aortic Mean Gradient First Month & 49 & $12.6 \pm 5.0$ & 11 & 6 & 27 & 55 & $8.3 \pm 5.1$ & 8 & 0 & 28 & $<.001 *$ \\
Aortic Mean Gradient Sixth Month & 48 & $12.6 \pm 7.8$ & 10.5 & 6 & 50 & 48 & $8.2 \pm 4.6$ & 8 & 0 & 28 & $<.001 *$ \\
\hline
\end{tabular}

*Mann-Whitney $\cup$ Test

a higher risk of death over the period of 6 months in both the TAVR group and surgery group. In particular, vascular complications in the TAVR group were an important cause of prolonged hospital discharge (10 patients; $17.9 \%)$. There were no vascular complications in the SAVR group. Perclose ProGlide ${ }^{\circledR}$ suture mediated closure system was used in all 10 patients with vascular complications. Percutaneous entry of the devices during TAVR disrupts the integrity of the vascular structure. If there is dense plaque and calcification in the vascular structure, this system could not provide effective suturing. Also, in obese patients who used the device for the first time, the closure system did not work because it could not be placed in the right place. In all of these patients, the vessel was explored and repaired.

Arrhythmia and block developed after both procedures and postoperative arrhythmia significantly was higher in the TAVR group (SAVR: $3.7 \%$, TAVR: 17.5\%). In the TAVR group, atrioventricular block developed in 3 patients, atrioventricular full block developed in 3 patients, supraventricular tachycardia developed in 2 patients, and ventricular fibrillation developed in 2 patients. In the SAVR group, one patient developed atrioventricular block and another patient developed supraventricular tachycardia. New onset atrial fibrillation within 30 days after the procedure was similar in the TAVR and SAVR groups (22.2 versus 21.4). After AF, 9 patients in both groups were returned to normal sinus rhythm with antiarrhythmic medical treatment and synchronous cardioversion.

During the 6-month follow-up period, hospital readmission in both groups took place for a variety of reasons (SAVR: 10 patients, TAVR: 23 patients). Of the 10 patients in the SAVR group, 6 presented with melena, one with nasal bleeding, one with sternum dehiscence, one with dyspnea, and one with atrial fibrillation. Four of these patients were admitted within the first week, 3 patients between the first week and first month, and 3 patients between the first and sixth months. Of the 23 patients readmitted to the hospital in the TAVR group, 13 had dsypnea, one presented with temporary ischemic attacks, 6 patients had angina, and 3 patients presented with atrial fibrillation. In the TAVR group, 4 patients were admitted within the first week, 9 patients between the first week and first month, and 10 patients between the first and sixth months.

After the procedures, revision was necessary for 1 patient in the SAVR group and 3 patients in the TAVR group. In the
SAVR group, it was operated due to the drainage of $450 \mathrm{cc}$ in the first 3 hours. In the TAVR group, 3 patients developed pericardial tamponade. Repair was needed for aortic bleeding in 1 patient and ventricular bleeding in 2 patients. There was no significant difference between the 2 groups.

The need for permanent pacemakers was higher in the TAVR group (1 patient versus 12 patients). After the SAVR, although 6 patients needed temporary pacemakers after surgery, only 1 needed a permanent pacemaker. In this group of patients, 1 patient had complete atrioventricular block, and 4 patients had bradycardia. On postoperative Day 1, he started working in a normal sinus rhythm (heart rate $>60$ ), and there was no need for a temporary pacemaker. In the TAVR group, 12 patients (3 patients with atrioventricular full block, 3 patients with atrioventricular block, and 6 patients with bradycardia) needed a pacemaker. Four patients with bradycardia returned to a normal sinus rhythm. However, the remaining 2 patients did not return to normal sinus rhythm. At the end of the first week, permanent pacemakers were inserted (Table 2).

At the postoperative first month, $62.5 \%$ of patients in the SAVR group were NYHA Class I and $37.5 \%$ were NYHA Class II. In the TAVR group, $24.1 \%$ of the individuals were Class I, $44.4 \%$ were Class II, $29.6 \%$ were Class III, and $1.9 \%$ were Class IV.

In the SAVR group, NYHA Class I was higher in the postoperative first month than in the TAVR group. In the TAVR group, it is seen that there is more NYHA Class III than in the SAVR group. At the postoperative sixth month, $91.7 \%$ of the SAVR group and $34.0 \%$ of the TAVR group were NYHA Class I. At the end of the sixth month, those in the SAVR group were more likely to be ranked in NYHA Class I than those in the TAVR group (Table 3).

Echocardiographic findings: Transthoracic echocardiography (TTE) was performed with GE Healthcare Vivid ${ }^{\mathrm{TM}}$ T8 preoperatively at 1 and 6 months and postoperatively in all patients undergoing TAVR and SAVR. Ejection fraction, aortic valve diameter, mean and peak gradient in the aorta, and paravalvular leak were evaluated with TTE from baseline to 6 months. In the SAVR patients, we implanted a mechanical valve with patients (6 patients no: 19; 23 patients no: $21 ; 20$ patients no: $23 ; 4$ patients no: 25 , and 1 patient no: 27). In the TAVR group, we implanted patients with a valve (23 patients no: 26; 26 patients no: 29; and 8 patients no: 31 ).

In the case of both procedures, from baseline to 6 months, the aortic-valve areas and left ventricular ejection fraction 
Table 5. Comparison of aortic insufficiency in SAVR and TAVR patients after procedure with TTE

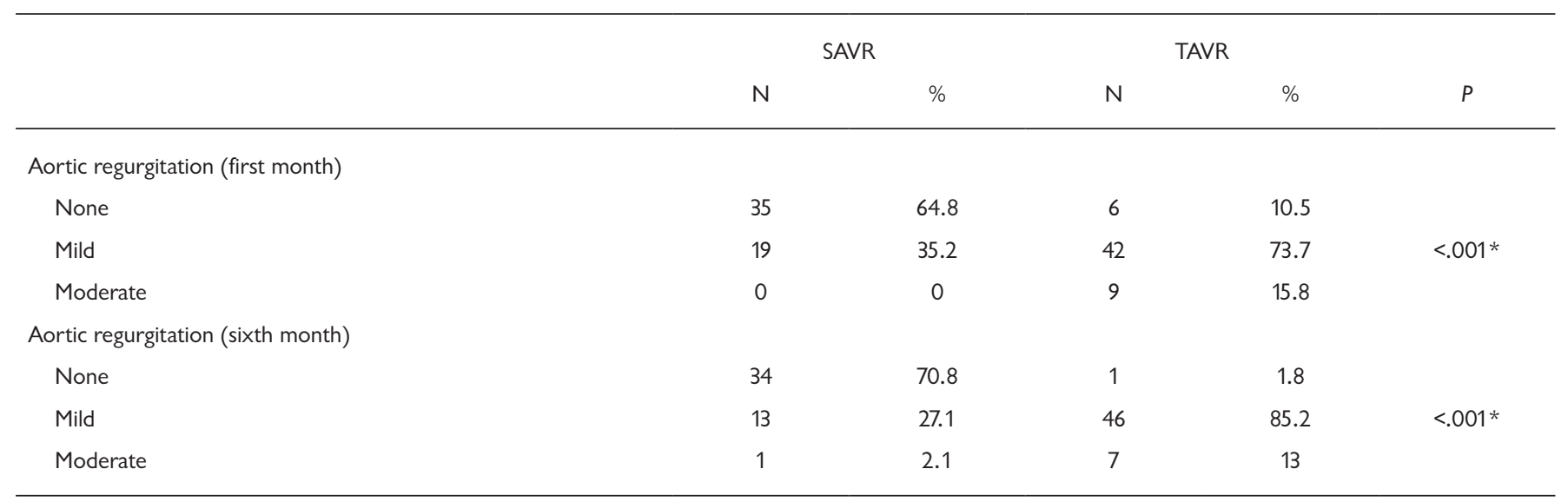

Chi-Square test was applied. *Fisher's exact test

significantly increased and the mean aortic valve gradients significantly decreased. In our study, mean gradient was higher in the SAVR group at the end of sixth month $(12.6 \pm$ $7.8 \mathrm{mmHg}$ versus $8.2 \pm 4.6$ ) (Table 4 ).

The improvements in aortic valve areas and gradients at all time points significantly were greater after TAVR. The frequency and severity of paravalvular aortic regurgitation were greater after TAVR. In the TAVR group at 1 month, mild paravalvular aortic regurgitation was observed, according to the standard classification scheme in $73.7 \%$ of patients and moderate paravalvular aortic regurgitation in $15.8 \%$ of patients. In the SAVR group at 6 months, mild paravalvular aortic regurgitation was observed, according to the standard classification scheme in $35.2 \%$ of patients, and moderate paravalvular aortic regurgitation in $0 \%(P<.001)$. In the TAVR group at 6 months, mild paravalvular aortic regurgitation was observed, according to the standard classification scheme in $85.2 \%$ of patients and moderate paravalvular aortic regurgitation was observed in $13 \%$ of patients. In the SAVR group at 6 months, mild paravalvular aortic regurgitation was observed, according to the standard classification scheme in $27.1 \%$ of patients and moderate paravalvular aortic regurgitation was observed in $2.1 \%$ of patients $(P<.001)$ (Table 5$)$.

The reason for high PVL in SAVR patients was evaluated as washing jet. Valve sizes were placed in accordance with the patient's body mass index. We understand there was valve prosthesis-patient mismatch due to the decrease in gradient rates in follow-up TTEs.

\section{DISCUSSION}

Although our study was in parallel with the results of many studies, different results were present in the parameters we obtained. The valve area index should be below $0.9 \mathrm{~cm}^{2} / \mathrm{m}^{2}$ in patients undergoing surgery. Although the valve index is normal, patients may still have gradient [Fernandez 1996]. In our study, mean and peak gradient was observed similar to other studies. There is less gradient in the valves used in TAVR than in the current central laminar form [Emery 1979]. Our post-procedure gradient results in patients treated with TAVR were similar to other studies.

In the PARTNER study, neurological events occurred in 31 of TAVR patients and 16 SAVR patients. In our study, cerebrovascular accidents occurred in 1 SAVR patient and 3 TAVR patients. In the TAVR patients, 12 had cerebrovascular events after the procedure; 6 patients in the SAVR group had cerebrovascular events after the procedure. In our study, a procedure-related cerebrovascular event occurred in 1 patient, and there was no statistically significant difference. All cerebrovascular events were of ischemic origin. Therefore, atherosclerotic plaque or air embolism were considered possible causes, during the procedure. Attention to these situations during both procedures can minimize cerebrovascular risk. In the PARTNER study, major vascular complications developed in 40 patients $(11.6 \%)$ in the TAVR group and in 13 patients (3.8\%) in the SAVR group. In our study, while no patients had vascular complications in the SAVR group, there were 10 patients in the TAVR group. Similar to our study, peripheral vascular complications were higher in the TAVR group. The most important reason here is that more peripheral vascular complications emerged both in our study and in the other studies at new centers. In addition, these results should be taken into consideration in newly developed devices for TAVR. Considering the need for a new pacemaker, it was observed that there were 21 (6.4\%) patients in the TAVR group and 17 (5.3\%) patients in the SAVR group. In our study, 12 (21.4\%) patients in the TAVR group and 1 (1.9\%) patient in the SAVR group needed a permanent pacemaker. While there was no significant difference in the PARTNER study, in our study, the need for a statistically significant pacemaker was higher in the TAVR group. The reason for this situation was thought to be due to oversized balloon dilatation after the valve was implanted during TAVR. In the PARTNER study, renal failure was observed in $18(5.4 \%)$ patients in the TAVR group and in $20(6.5 \%)$ 
patients in the SAVR group. In our study, it developed in 2 (3.6\%) patients in the TAVR group and $4(7.4 \%)$ patients in the SAVR group. Looking at both the PARTNER and our study, there was no significant difference in either group [Mack 2015].

Considering vascular complications in the study of Gustav Horsted et al, 8 (5.6\%) patients developed in the TAVR group and $2(1.5 \%)$ patients in the SAVR group. In our study, vascular complications were higher in the TAVR group. Although it was not statistically significant in the study of Gustav Horsted, it was seen that the number was higher in the TAVR group. Acute kidney injury was found to be in $1(0.7 \%)$ patient in the TAVR group and $9(6.7 \%)$ patients in the surgical group. There also was significantly more renal failure in the surgical group. In our study, 2 (3.6\%) patients in the TAVR group and $4(7.4 \%)$ patients in the SAVR group developed acute kidney injury. When we examined cerebrovascular events, it was seen that there were 4 patients ( 2 patients TIA, 2 patients stroke) in the TAVR group and 4 (3\%) patients (all strokes) in the SAVR group. In our study, 3 patients in the TAVR group and 1 patient in the SAVR group had cerebrovascular events. There was no significant difference in either study. In Gustav Horsted's study, 24 (16.9\%) patients in the TAVR group and 77 (57.8\%) patients in the SAVR group developed atrial fibrillation. In our study, atrial fibrillation developed in 12 (22.2\% vs $21.4 \%$ ) patients in both groups. There was no statistically significant difference in both studies. In Gustav Horsted's study, the need for permanent pacemakers was needed in 46 (34.1\%) patients in the TAVR group and in $2(1.6 \%)$ patients in the SAVR group. Similarly, in our study, a pacemaker was needed in $1(1.9 \%)$ patient in the SAVR group and $12(21.4 \%)$ patients in the TAVI group. In the study of Gustav Horsted, when patients were examined at the end of the first year, NYHA Class II patients were $29.5 \%$ in the TAVR group and $15 \%$ in the SAVR group, according to NYHA classification. Moderate dyspnea was higher in the TAVR group. In our study, at the end of 6 months, 18 patients in the SAVR group and 40 patients in the TAVR group were in NYHA Class II. This was statistically significant in both studies. In the study of Gustav Horsted, $71.1 \%$ of patients in the TAVR group had moderate and advanced aortic insufficiency at the end of the first year. In the SAVR group, there was $17.7 \%$ of middleand advanced-aortic insufficiency. In our study, at the end of the sixth month, mild (27.1\%) and moderate $(2.1 \%)$ aortic insufficiency was found in $29.2 \%$ patients in the SAVR group. In the TAVR group, $98.2 \%$ had mild (85.2\%) and moderate $(13 \%)$ aortic insufficiency. Both our study and that of Gustav Horsted showed aortic insufficiency was higher in the TAVR group [Gustav Horsted 2015].

In the study of Leon et al., 10 patients in the TAVR group and 8 patients in the SAVR group died during the procedure or within 3 days. In our study, 5 patients in the SAVR group and 3 in TAVR group died within 6 months. There is no statistical difference in both studies. In the first month, 64 patients (9 of all patients TIA) in the TAVR group, 65 patients (4 of all patients TIA) in the SAVR group had cerebrovascular events. At the end of the first year, 99 patients (23 of all patients TIA) in the TAVR group and 93 patients (16 of all patients TIA) in the SAVR group had cerebrovascular events. In our study, 3 patients in the TAVR group and 1 patient in the SAVR group had cerebrovascular events. There is no statistical difference in both studies. At the end of the first year, 142 (14.8\%) patients in the TAVI group and 135 (14.7\%) patients in the SAVR group were rehospitalized. In our study, 23 patients in the TAVR group and 10 patients in the SAVR group were rehospitalized. There is no statistically significant difference. Acute renal failure developed in 13 patients in the TAVR group and in 31 patients in the SAVR group. Similar to our study, there was no significant difference between the 2 groups. After the procedure, permanent pacemakers were implanted in 85 patients in the TAVR group and 68 patients in the SAVR group. In our study, 1 patient in the SAVR group and 12 patients in the TAVR group required a permanent pacemaker. It is statistically significant, and we obtained different results from the other study. Atrial fibrillation occurred in 91 patients in the TAVR group and 265 patients in the SAVR group. Statistically, this was significant. In our study, atrial fibrillation occurred in 12 patients. Unlike our study, atrial fibrillation was more common in the SAVR group [Leon 2016].

In the study of Smith et al, 84 patients in the TAVR group and 89 patients in the SAVR group died within one year. In our study, 5 patients in the SAVR group and 3 in TAVR group died within 6 months. There is no statistical difference in both studies. In the first month, 19 patients ( 3 of all patients TIA) in the TAVR group, 8 patients ( 1 patient TIA) in the SAVR group. At the end of the first year, 27 patients (7 of all patients TIA) in the TAVR group, and 13 patients (4 of all patients TIA) in the SAVR group had cerebrovascular events. In our study, 3 patients in the TAVR group and 1 patient in the SAVR group had cerebrovascular events. There is no statistical difference in both studies. At the end of the first year, $58(18.2 \%)$ patients in the TAVR group and 45 (15.5\%) patients in the SAVR group were rehospitalized. In our study, 23 patients in the TAVR group and 10 patients in the SAVR group were rehospitalized. There is no statistical significant difference. Acute renal failure developed in 4 patients in the TAVR group and in 4 patients in the SAVR group. Similar to our study, there was no significant difference between the 2 groups. After the procedure, 13 patients in the TAVR group and 12 patients in the SAVR group were implanted with permanent pacemakers. In our study, 1 patient in the SAVR group and 12 patients in the TAVR group required permanent pacemakers. It is statistically significant, and we obtained different results from the other study. In terms of vascular complications, 59 patients in the TAVR group and 13 patients in the SAVR group developed vascular complications. Statistically, similar results were obtained with our study.

While 30 patients experienced atrial fibrillation in the TAVR group, there were 56 patients who experienced atrial fibrillation in the SAVR group. In our study, atrial fibrillation occurred in 12 patients. There was no significant difference in either group. In the study of Smith et al, the mean gradient was higher in the SAVR group as a result of TTE at the end of the first year $(10.2 \pm 4.3 \mathrm{~mm} \mathrm{Hg}$ versus $11.5 \pm 5.4$ $\mathrm{mm} \mathrm{Hg}$ ). But it was not statistically significant. The moderate and severe paravalvular leak was more common in the TAVR 
group than on the 30th day (12.2\% versus $0.9 \%)$ and the first year $(6.8 \%$ versus $1.9 \%)$. In our study, mean gradient was higher in the SAVR group at the end of sixth month. (12.6 \pm $7.8 \mathrm{mmHg}$ versus $8.2 \pm 4.6$ ) both studies had similar results. In our study, both the first month and sixth month NHYA were evaluated. The patients in the SAVR group had Class I symptoms, while the number of patients with TAVR patients with Class III symptoms were high. In fact, no symptoms of Class III SAVR patients were seen in the sixth month. After TAVR, 24\% had Class III patients [Smith 2011].

In the study of Susheel et al, 114 patients in the TAVR group and 116 patients in the SAVR group died within 2 years. In our study, 5 patients in the SAVR group and 3 patients in the TAVR group died within 6 months. There is no statistical difference in both studies. In 2 years, 34 patients (10 of all patients TIA) in the TAVR group and 18 patients (5 of all patients TIA) in the SAVR group had cerebrovascular events. In our study, 3 patients in the TAVR group and 1 patient in the SAVR group had cerebrovascular events in 6 months. There is no statistical difference in both studies. At the end of the second year, 74 (24.7\%) patients in the TAVR group and 60 (21.7\%) patients in the SAVR group were rehospitalized. In our study, 23 patients in the TAVR group and 10 patients in the SAVR group were rehospitalized. There is no statistically significant difference. Acute renal failure developed in 20 patients in the TAVR group and in 21 patients in the SAVR group. Similar to our study, there was no significant difference between the 2 groups. After the procedure, 23 (7.2\%) patients in the TAVR group and $19(6.4 \%)$ patients in the SAVR group were implanted with permanent pacemakers. In our study, 1 patient in the SAVR group and 12 patients in the TAVR group required permanent pacemakers. No statistical difference was found. Susheel et al found that TAVR patients had more insufficiency and moderate paravalvular insufficiency increased lateterm mortality at the two-year follow up [Susheel 2012]. In our study, there were 19 mild insufficiency in SAVR patients and 42 mild insufficiency in TAVR patients in the first month. In the sixth month, there were 13 mild and 1 moderate insufficiency in the SAVR patients; 46 mild and 7 moderate aortic insufficiency in the TAVR patients [Susheel 2012].

In the study of Daubert et al, in the TAVR group mean gradient is $11.5 \pm 5.4$ immediately after the procedure was measured as mean gradient is $11.0 \pm 6.3$ after 5 years. In the SAVR group, mean gradient is $12.1 \pm 5.0$ immediately after procedure was measured as mean gradient is $10.6 \pm 5.5$ after 5 years. But it was not statistically significant. In the aortic insufficiency TAVR group, it was found more in the first measurements after the procedure compared to SAVR. At the end of the fifth year, aortic insufficiency is higher. In our study, aortic insufficiency is higher in the TAVR group [Daubert 2017].

Our study has several limitations. First, it was a single center and retrospective study. There was a significant difference between the age groups, even if the patients were selected for moderate and high risk. TAVR cases are the first cases that were performed in our center. Therefore, patients had longer hospitalizations, complications (vascular complication contrast nephropathy), and more frequent follow-up visits. Finally, the follow-up period was 6 months. Longer follow up of patients should be done.

In conclusion, we have shown that in patients with aortic stenosis who are at high/intermediate risk for operative complications and death, surgical aortic-valve replacement and TAVR were associated with similar mortality at 6 months. Our findings indicate that transcatheter replacement is an alternative to surgical replacement in a well-chosen, high-risk subgroup of patients with aortic stenosis. Lastly, according to SAVR, patients admitted to hospital more frequently after TAVR were associated with insufficiency of the aortic valve after the procedure.

\section{REFERENCES}

Daubert MA, Weissman NJ, Hahn RT, Pibarot P, Parvataneni R, Mack MJ, Douglas PS. 2017. Long-Term Valve Performance of TAVR and SAVR. JACC: Cardiovascular Imaging, 10(1), 15-25.

Emery RW, Nicoloff DM, Fisher M, et al. 1979. St. Jude Medical cardiac valve prosthesis: in vitro studies. J Thorac Cardiovasc Surg 78:269-76.

Fernandez J,Chen C, et al. 1996. Predictive value of prosthetic valve area index for early and late clinical results after valve replacement with the $\mathrm{St}$ Jude Medical valve prosthesis. Circulation 94:109-12.

Gustav Horsted H, Steinbrüchel DA, et al. 2015. Transcatheter versus surgical aortic valve replacement in patients with severe aortic valve stenosis, J Am Coll Cardiol 65:2184-94.

Leon MB, Smith CR, et al. 2016. Transcatheter or surgival aortic valve replacement in intermediate risk patients, N Engl J Med 374:1609-20.

Mack MJ, Leon MB, Smith CR, et al. 2015. 5-year outcomes of transcatheter aortic valve replacement or surgical aortic valve replacement for high surgical risk patients with aortic stenosis (PARTNER 1): a randomised controlled trial. Lancet 385: 2477-84.

Nishimura RA. 2002. Low-Output, Low-Gradient Aortic Stenosis in Patients With Depressed Left Ventricular Systolic Function. Circulation. 106:770-772.

O'Brien SM, Shahian DM, Filardo G, et al. 2009. The Society of Thoracic Surgeons 2008 cardiac surgery risk models. 2. Isolated valve surgery. Ann Thorac Surg 88:Suppl 1:S23-42.

Smith CR, Leon MB, et al. 2011. Transcatheter versus Surgical aorticvalve replacement in high-rish patients, N Eng J Med 364:2187-98.

Smith CR, Leon MB, Mack MJ. 2011. Transcatheter versus Surgical Aortic-Valve Replacement in High-Risk Patients. N Engl J Med 364:2187-2198.

Susheel K, Mathew R, et al. 2012. Two-Year outcomes after transcatheter or surgical aortic valve replacement, N Engl J Med 336:1686-95. 